\title{
The CMS electron and photon trigger for the LHC Run 2
}

\author{
Andrea Beschi ${ }^{* \dagger}$ \\ Università \& INFN, Milano-Bicocca (IT) \\ E-mail: andrea.beschi@cern.ch
}

The Compact Muon Solenoid (CMS) experiment implements a sophisticated two-level triggering system composed of a custom-designed hardware Level-1 trigger and a software High-LevelTrigger. A new Level-1 trigger architecture with improved performance is now being used to maintain thresholds compatible with the CMS physics program despite the challenging luminosity conditions experienced during Run II. We present the performance of the upgraded CMS electron and photon trigger. The calorimeter trigger system plays a central role in achieving the ambitious physics program of Run II. The upgraded trigger uses the granularity of the calorimeters to optimally reconstruct the electromagnetic trigger objects. The performance of the new trigger system will be presented, based on proton-proton collision data collected in Run II. The selection techniques used to trigger efficiently will be presented, along with the strategies employed to guarantee efficient triggering for new resonances and other new physics signals involving electron/photon final states.

The European Physical Society Conference on High Energy Physics

5-12 July

Venice, Italy

* Speaker.

†n behalf of the CMS Collaboration. 


\section{Introduction}

The running conditions of the Large Hadron Collider (LHC) Run II represents a new challenge for the CMS experiment [1], since the foreseen instantaneous luminosity is up to $\mathscr{L}=$ $2 \cdot 10^{34} \mathrm{~cm}^{-2} \mathrm{~s}^{-1}$, twice the nominal design value of the LHC. In this luminosity scenario LHC will deliver up to 60 proton-proton collisions per bunch crossing (pile-up). The Level-1 (L1) trigger system architecture underwent a major improvement in 2016 [2]. The trigger algorithms were retuned in 2017 to fully exploit the potential of the system and to maintain transverse momentum $\left(\mathrm{p}_{T}\right.$ ) thresholds [2] compatible with the CMS physics program despite the new running conditions. The new algorithm for electron and photon $(\mathrm{e} / \gamma)$ triggers and its performance with early 2017 data are described in the following.

\section{Identification of $\mathrm{e} / \gamma$ candidates}

The L1 e/ $\gamma$ trigger algorithm $[3,4]$ relies on energy deposits in the electromagnetic calorimeter (ECAL), while the energy in the hadronic calorimeter (HCAL) is used for jet-rejection and isolation. The calorimeters are segmented in the $(\eta, \phi)$ directions in trigger towers (TT). The clustering algorithm dynamically aggregates energy deposits in neighbouring TTs around a seed tower which constitutes a local energy maxima, as shown in Fig. 1 (left). The dynamic clustering efficiently recovers energy losses due to bremsstrahlung radiation improving the energy resolution. The energy of the e $/ \gamma$ candidate corresponds to the sum of the energy of the TTs in the cluster. The L1 clustering algorithm includes an energy calibration applied in bins of pseudorapidity and the topological shape of the candidate. To discriminate against jets, a selection is applied on the ratio between the energy deposited in the HCAL and in the corresponding ECAL tower $(\mathrm{H} / \mathrm{E})$. A shape veto further rejects candidates with jet-like shapes, while retaining $99.5 \%$ of true e $/ \gamma$ candidates. Figure 1 (right) shows examples of the shape veto functionality. Additionally events are considered isolated if the energy deposited in a $6 \times 9(\eta, \phi)$ TT window around the cluster seed (Fig. 1, centre) is below a fixed threshold. This threshold is adjusted depending on the pseudorapidity, energy of the candidate and the estimated pile-up (from the number of TTs with a non-zero energy deposition).

\section{Performance of the algorithm}

The algorithm performance was measured with data collected in 2017 and compared with data collected in 2016 [5]. The two datasets used for performance comparison have an average pile-up of 24 collisions per bunch crossing. The algorithm improves the H/E selection and retunes the isolation threshold. Energy calibrations were updated to match the change in detector response. Figure 2 (left) shows the spread of the energy ratio between the offline reconstructed electron energy and the L1 energy in bins of pseudorapidity of the electron supercluster for 2016 (red) and 2017 (blue). The optimized calibration provided an energy resolution similar to that of 2016 in the ECAL barrel and better than the resolution of 2016 in the ECAL endcaps. The improved H/E selection increases the rejection of jets faking electrons, reducing the trigger rate by about $20 \%$ without any significant efficiency loss for electrons and photons. The H/E selection is applied not 


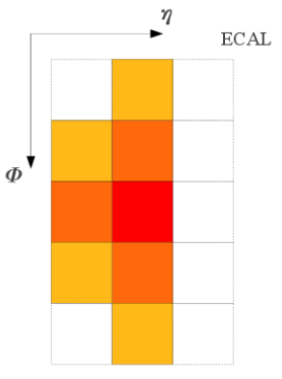

Seed tower First neighbours

Second neighbours

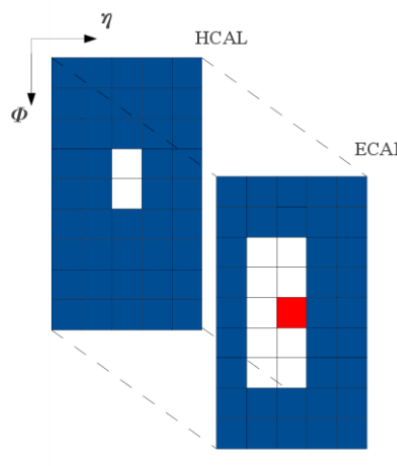

Isolation region

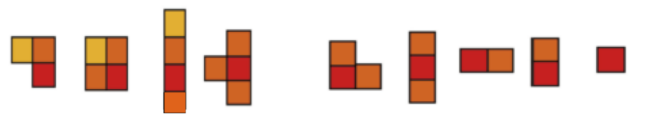

Examples of $e / v$ like cluster shapes

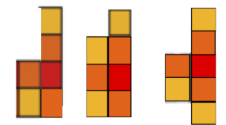

Examples of jet like cluster shapes

Figure 1: Left: an example of how the dynamic clustering merges different trigger towers (TTs) in a cluster. Centre: the illustration of the isolation region (blue) around a cluster seeded by the red trigger tower. Right: examples of cluster shapes for e/ $\gamma$-like candidates and jet-like candidates $[3,4]$.
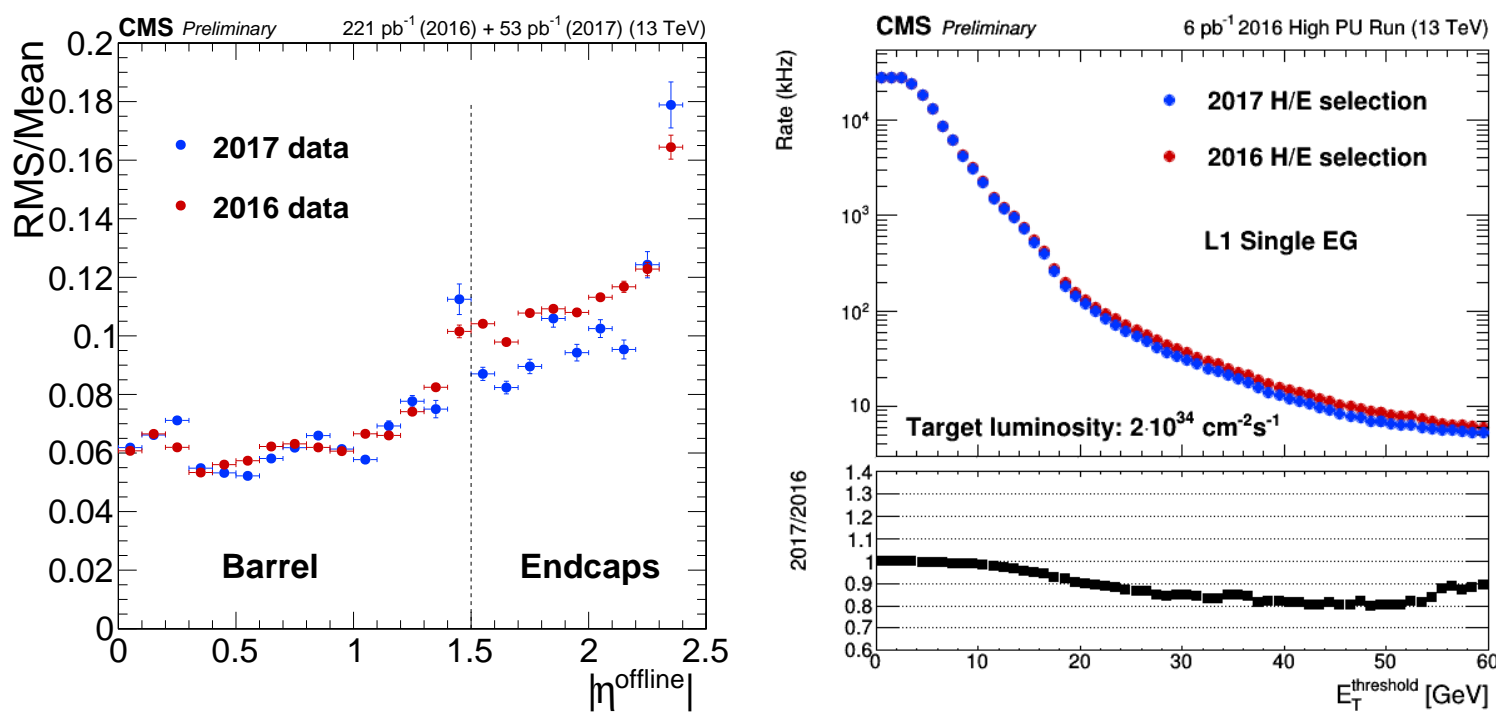

Figure 2: Left: Level-1 e/ $\gamma$ trigger resolution with respect to offline electrons as a function of the pseudorapidity $\eta$ of the electron supercluster for 2016 (red) [5] and 2017 (blue) data. The two datasets have an average pile-up of 24 vertices per bunch crossing. Right: Expected rate for e $/ \gamma$ trigger as a function of the $\mathrm{p}_{T}$ threshold for a luminosity of $2 \cdot 10^{34} \mathrm{~cm}^{-2} s^{-1}$. The plot shows a comparison applying a selection on e/ $\gamma$ candidates based on the ratio between energy deposited in the HCAL and in the ECAL (H/E) with 2016 algorithm (red) [5] and the 2017 updated H/E criteria (blue). The bottom plot is the ratio of the 2017 rate to the 2016 rate. The collected data are re-emulated with the 2017 trigger conditions applying the two different $\mathrm{H} / \mathrm{E}$ criteria. 
only in the seed TT as in 2016 but also in a $3 \times 3$ TTs window around the seed. Figure 2 (right) compares the predicted rate for an instantaneous luminosity of $\mathscr{L}=2 \cdot 10^{34} \mathrm{~cm}^{-2} \mathrm{~s}^{-1}$ with 2016 $\mathrm{H} / \mathrm{E}$ selection and the criteria used in 2017. To further increase the rejection of energy deposits due to pile-up, the isolation threshold has been reoptimized. Figure 3 (left) shows the efficiency of the $\mathrm{L} 1 \mathrm{e} / \gamma$ algorithm as a function of the number of reconstructed primary vertices. The efficiency for electrons with offline-reconstructed $\mathrm{p}_{T}>35 \mathrm{GeV}$ is computed on data. The new isolation selection ensures higher efficiency than 2016 independently of the pile-up. Similarly Fig. 3 (right) shows the efficiency of the e/ $\gamma$ trigger as a function of the transverse energy of the offline reconstructed electron. The redesigned isolation and the $\mathrm{H} / \mathrm{E}$ criteria increase the efficiency on e/ $\gamma$ candidates and reduce the rate due to jets faking electrons, resulting in a lower $\mathrm{p}_{T}$ threshold and a sharper turn-on. As an extra handle to preserve the $\mathrm{p}_{T}$ thresholds compatible with the CMS physics program, the $\mathrm{L} 1$ trigger implements sophisticated algorithms targeting $\mathrm{Z} \rightarrow$ ee decays and $\mathrm{W} \rightarrow \mathrm{e} v$ [6]. The $\mathrm{Z}$ boson is selected requiring the presence of two e $/ \gamma$ candidates with invariant mass compatible with a $\mathrm{Z}$ boson, the $\mathrm{W}$ boson trigger relies on the presence of an e/ $\gamma$ candidate and missing transverse momentum $p_{T}^{\text {miss }}$, defined as the magnitude of the projection onto the plane perpendicular to the beam axis of the negative vector sum of the momenta of all reconstructed objects in an event.
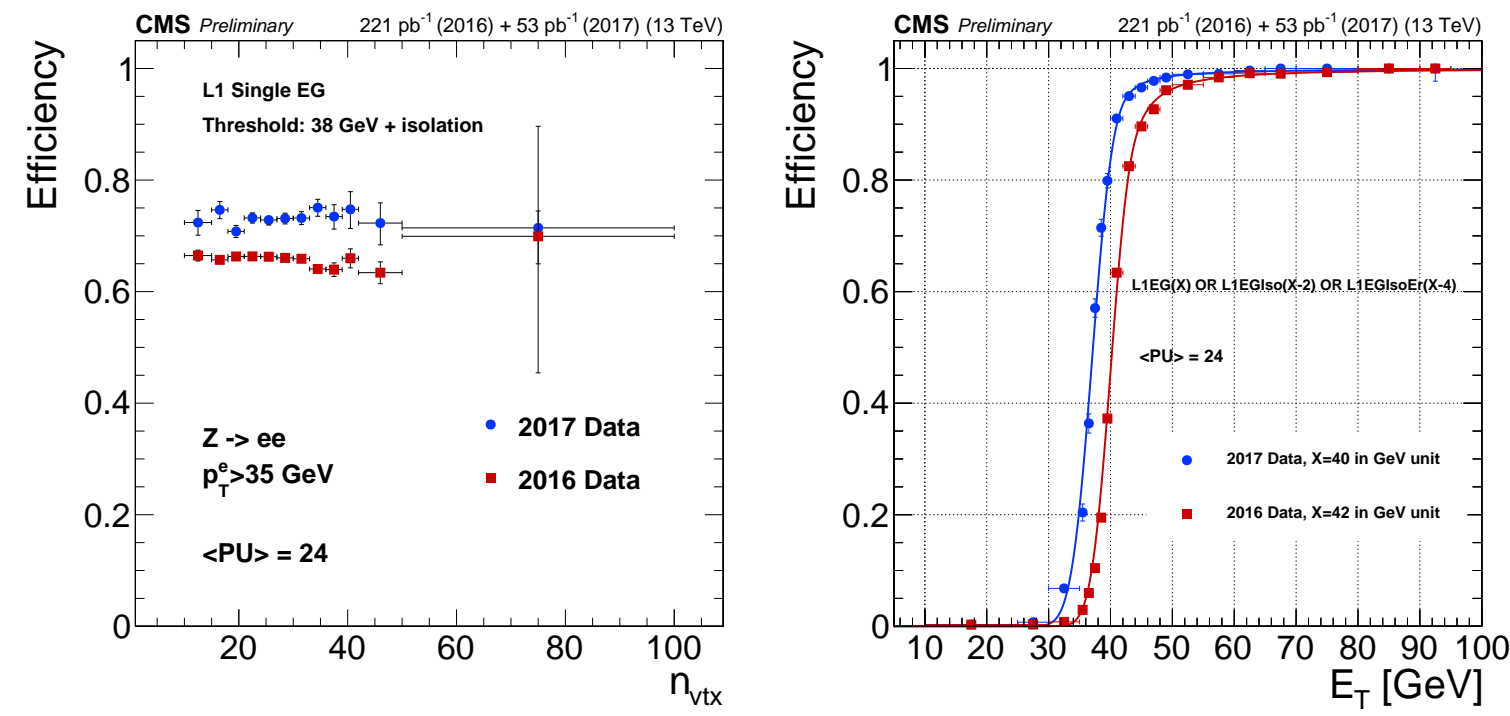

Figure 3: Left: Level-1 e/ $\gamma$ trigger efficiency requiring isolated e $/ \gamma$ candidates with $\mathrm{p}_{T}>38 \mathrm{GeV}$ as a function of the number of offline reconstructed vertices for 2016 (red) [5] and 2017 (blue) data. The efficiency is measured on a sample of $\mathrm{Z} \rightarrow e e$ requiring the offline reconstructed probe electron to have a transverse energy greater than $35 \mathrm{GeV}$. The two curves correspond to the same trigger rate. Right: L1 trigger efficiency curve for an $\mathrm{e} / \gamma$ object as a function of the offline reconstructed supercluster transverse energy $\mathrm{E}_{T}$ with the 2017 algorithm (blue) and the 2016 algorithm (red) [5] measured on data. A geometrical matching between the electron supercluster and the L1 candidate is applied. The efficiency is drawn for the Single EG strategy present at L1: e/ $\gamma$ with $\mathrm{p}_{T}>X \mathrm{GeV}$ without isolation requirements $(\mathrm{EG}) \mathrm{OR}$ e/ $\gamma$ with $\mathrm{p}_{T}>(X-2) \mathrm{GeV}$ and isolation requirement (EGIso) OR e/ $\gamma$ with $\mathrm{p}_{T}>(X-4) \mathrm{GeV}$, isolation requirement and pseudorapidity $|\eta|<2.1$ (EGIsoEr). The two curves are drawn for the thresholds $\mathrm{X}$ ensuring the same trigger rate. 


\section{Summary}

The L1 trigger e/ $\gamma$ algorithm was optimized to fully exploit the potential of the trigger system and to ensure an efficient trigger for electrons and photons at instantaneous luminosities up to $\mathscr{L}=2 \cdot 10^{34} \mathrm{~cm}^{-2} \mathrm{~s}^{-1}$. After the optimization of the L1 trigger algorithm, the $\mathrm{p}_{T}$ thresholds of the e/ $\gamma$ trigger are compatible with the CMS physics program and with sharper turn-on for the same trigger rate.

\section{References}

[1] CMS Collaboration, S. Chatrchyan et al., The CMS Experiment at the CERN LHC, JINST 3 (2008) S08004.

[2] CMS Collaboration, A. Tapper and D. Acosta, CMS Technical Design Report for the Level-1 Trigger Upgrade, No. CERN-LHCC-2013-011. CMS-TDR-12, Jun, 2013, https://cds.cern.ch/record/1556311.

[3] CMS Collaboration, N. Dev et al., The CMS Level-1 electron and photon trigger: for Run II of LHC, in Proceedings, Topical Workshop on Electronics for Particle Physics (TWEPP 2016): Karlsruhe, Germany, vol. 12, p. C02014, 2017, DOI.

[4] CMS Collaboration, J.-B. Sauvan, Performance and upgrade of the CMS electron and photon trigger for Run 2, in Proceedings of 16th International Conference on Calorimetry in High Energy Physics (CALOR 2014): Giessen, Germany, April 6-11, 2014, vol. 587, p. 012021, 2015, DOI.

[5] CMS Collaboration, A. Zabi et al., The CMS Level-1 Calorimeter Trigger for the LHC Run II, in Proceedings, Topical Workshop on Electronics for Particle Physics (TWEPP 2016): Karlsruhe, Germany, vol. 12, p. C01065, 2017, DOI.

[6] CMS Collaboration, C. Wulz, Data analysis at CMS Level-1 Trigger level: Migrating complex selection algorithms from offline analysis and High-Level trigger to the trigger electronics, PoS EPS-HEP (2017) . 